\title{
THE IMPACT OF INFLUENZA ON GENERAL PRACTICE
}

\author{
R. J. F. H. Pinsent, M.A., M.D., M.R.C.S., L.R.C.P. \\ General Practitioner, Birmingham
}

I waS glad to see the word 'elsewhere' in the title of this symposium for we cannot consider influenza outside the context in which it occurs, and where it is first observed by the family doctor. Influenza is a general-practitioner illness par excellence and as such is treated almost wholly without reference to hospital. Doctor and patient alike regard it as a condition to be managed at home, and do not associate uncomplicated influenza with hospitals at all.

Let us first look at influenza as the patient sees it. The word has entered the popular vocabulary and is frequently used, more often inaccurately than correctly. It is a pity that the word 'flu' is so easy to pronounce. Influenza is 'news' and the public regard for and reaction to it is conditioned by the Press and by other means of mass communication. Patients like to think that, as a result of this, they know all about it and they devise their own definitions for 'gastric flu', '24-hour flu', even 'muscular flu' as well as their own bizarre treatments. Thus public opinion, as well as the threshold of tolerance of illness by the individual, determines whether or no the doctor will be consulted rather than the chemist on the corner. It is at the point at which the general practitioner is consulted that influenza becomes a medical problem.

The first problem facing the family doctor is to do what the patient has already done, to define for himself the pattern of the 'influenza' with which he is presented by consecutive epidemics. $\mathrm{He}$ builds this up from a mental 'identi-kit' of symptoms and signs which increases in value with his experience. As the clinical signs and presentation vary from one epidemic of influenza to another so must the definition which he creates for himself and on which he bases his management and care. This definition is a personal one, and may not be the same as that of the next general practitioner. However, agreement on definitions in this field is hard to come by even among the experts.

Influenza must be distinguished from the remainder of the diseases of the respiratory tract which are commonly found in practice and undoubtedly early cases of true influenza are missed, in every epidemic, for they are unrecognized in the continuing sequence of respiratory disease. The emergence of a pattern, with wholebody symptoms and perhaps predilection for an age-group, is often the first indicator of an impending epidemic. The pattern may be a new one, or it may resemble those seen in the past. It becomes distinguishable from other respiratory disease patterns to which the practitioner is accustomed to attach the words 'bronchiolitis', 'acute bronchitis', 'febrile catarrh', etc. Whether it is possible to forecast an impending epidemic of true influenza by study of the mass of respiratory disease in the preceding weeks remains to be seen.

Fig. I represents consultations in respect of influenza as compared with consultations occasioned by other respiratory disease; an increase in the latter will be seen before the peak-abrups or slowly reached-caused by influenza itself Probably the early cases lie in this area, but sometimes there is a brief decrease in the amount of respiratory disease between the minor peak and the major one. The reason for this must arouse speculation. How far can we use this rise in total respiratory disease to predict the oncoming epidemic remains to be seen, but internal evidence from one affected practice can surely be made to become external advance evidence for another, a useful warning to other as yet unaffected practices.

In the early stages of an epidemic, as the pace of work whips up, the doctor recognizes the features peculiar to the particular kind of 'flu' with which he is dealing. Perhaps, as in 1957, schoolchildren are most affected at first, their parents subsequently in a second wave. On the other hand the outbreak may first affect teenagers and the doctor may expect to have to deal with some very severe epistaxes among them. If the pattern of the disease is imposed on the older age groups he will expect a high mortality from bronchopneumonia. In an epidemic of this kind he may admit one or two patients to hospital on account of their secondary illness. I personally cannot recall ever admitting a patient to hospital on account of influenza alone

Influenza demonstrates the iceberg phenomenon 


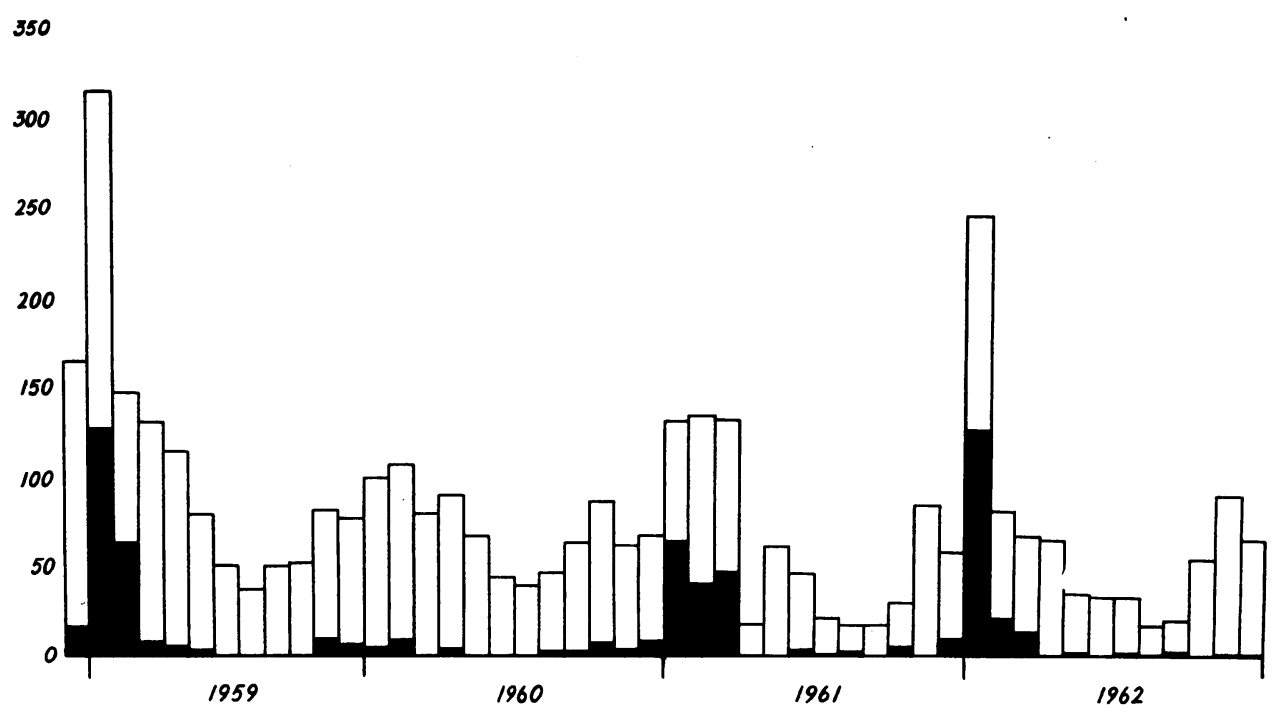

FIG. I.-Monthly consultations of patients with respiratory diseases and influenza.

particularly well-if admission to hospital is thought to be water level the main mass of the disease lies below. That part of the disease which is seen above the surface is not simple influenza but the primary disease complicated by something else, the complicating factor being almost invariably bacterial.

When the doctor has established his criteria for diagnosis the process of diagnosis is carried out rapidly and in each epidemic a standard treatment pattern is usually adopted. For various reasons I now tend to use paracetamol to relieve symptoms instead of aspirin, but treatment in the absence of complications is seldom more than symptomatic. Steam inhalations, flavoured with Friar's balsam if you like, will relieve discomfort in the chest while an accompanying sore throat benefits from local anæsthetic lozenges. The virus infection can only be treated by insistence on bed rest until the temperature has returned to normal for a day.

The onset of complications indicates the use of antibiotics; cough with sputum, particularly if purulent, calls for the antibiotic of choice which may be determined by the policy of the practice as much as the cultural characteristics and sensitivities of the organism. Wherever chest symptoms are a feature, a follow-up mass X-ray is routine, often revealing areas of consolidation even in the absence of clinical signs. These resolve before a repeat film is taken a month later.

The character of the epidemic may suggest a 'standard' time which the patient may expect to remain off work. This will influence his need for
National Health Insurance certificates and hences the number of attendances he makes on his doctor. One particular variable characteristic in influenza epidemics is the degree of debilityo following the illness. The influence of this on $\mathrm{a}^{\mathrm{C}}$ given epidemic only comes clear as the epidempiric goes on.

General practice, then, is the main field of conflict between the influenza viruses and the community and I sometimes feel that we do not make such good use of our resources as we might. $\overrightarrow{\vec{A}}$ Sometimes we see an epidemic of influenza coming afar off and though we may know that it means? extra work sooner or later we do little to prepareo for it. Not much can be done within a practice 'Chronic visiting' lists can be adjusted a little but 3 . the unreinforced practice has to brace itself and too prepare to improvise.

The number of doctors in general practice isô likely to be fewer for some years to come and $\mathrm{a}^{3}$ strong case can be made for giving relief to them? in epidemic periods by mobilizing medical and nursing resources not otherwise at the time ato full stretch. Only by so doing can observationah studies of the early disease be properly done? Would it be difficult for the Medical Officer of 0 Health to release some of his adminstrative and clinic staff for bedside and consulting room worko during an epidemic ? Could not the doctors seo released be attached to practices, and the arrange- $-\frac{\mathbb{D}}{}$ ment be made well in advance so that the practiti-? oners would know who was to be their firsto reserve? Could not someone-perhaps the M.O.H. again-keep a roster or register of 
married women doctors who could be called upon to do sessional work in practice during epidemics -and pay them for the sessions which they do ?

Planning is not only deficient on the local scale, but at national level also. The Public Health Laboratory Service operates, through general practitioners, an influenza spotting service, hoping thus to receive early warning of the type of 'flu' occurring in a given locality. We believe that an international influenza centre exists; but little information about its discoveries and activities percolates down to general practice.

Would it not be practicable, in the quiet periods between epidemics, to prepare a research plan in greater detail ? A notification system could perhaps be prepared in advance, to be put into operation by a central coordinating authority which would be able to distil epidemiological elixir from reports of presence or absence of a defined disease-pattern at a given time, backed up with clinical descriptions and material referred to the virologists at P.H.L.S. Laboratories. Epidemic after epidemic catches us unprepared, or sees us mesmerized into inactivity by its approach even when we are given warning.

Before we can properly assess the value of various immunizing procedures we must learn more about the natural history of influenza, and I submit that this is a task in which the clinician, the pathologist and the general practitioner will have to get together for constructive planning well in advance of the event; perhaps even before the next epidemic is due in the spring of 1964 .

Finally, few symposia of this kind have ever taken place without a definition of the disease under discussion. To remedy this deficiency I would like to suggest that influenza is a clinical condition observed in general practice, the complications of which are studied in hospital before the patient dies of a staphylococcal infection.'

\section{Discussion}

A QUESTIONER asked about chest signs in uncomplicated influenza; Professor STUART-HARris replied that the majority had clinical evidence of lower respiratory tract infection; in cases taken at random, about two out of three without previous chest disease have had chest signs.

Dr. HEarn remarked that influenza in hospital staff and among patients admitted for something else was usually pretty mild.

DR. FLEWETT described the influenza in a school for children with rheumatic heart disease as having been mild and almost sub-clinical; though as all the children had their temperatures taken routinely night and sometimes morning, transient fevers only found on one reading, up to $10 \mathrm{r}^{\circ} \mathrm{F}$., had been detected. Only a few children had been really ill. Sera from both the very mild and the severe cases alike provided laboratory evidence of influenza.

In answer to a question Professor STUART-Harris said that patients with staphylococcal lesions in themselves or their families were thought to be especially liable to staphylococcal pneumonia when they caught influenza. There appeared to be a special chain of in- fection from one case of staphylococcal disease to another.

Dr. Pinsent added that the family staph. infections were often not known and this would have to be ac study in the next epidemic.

DR. FLEwETT commented upon the difficulty which G.P.s had in getting specimens to the laboratory during epidemics. A collection system was needed, e.g. minivan to circulate among the practices.

Dr. Pinsent agreed, and said that blood collection was now easier; sterile packs were available. What was needed was a sterile syringe service and broth for taking garglings. He often saw patients too late; it was desirable to have specimens within 24 hours of the onset.

Dr. Hearn complained that similar difficulties occurred in hospitals. A real effort was required from the staff, during tides of heavy infection especially, to collect second sera.

DR. PINSENT complained that there was difficulty in collecting convalescent sera in general practice. Patients would not attend when they had recovered. It would be much easier if only one sample would do the job. 